\title{
Optimization of Focused Ion Beam-Tomography for Superconducting Electronics
}

\author{
Aric Sanders ${ }^{1}$, Anna Fox ${ }^{1}$, Paul Dresselhaus ${ }^{1}$, and Alexandra Curtin ${ }^{1}$ \\ 1. Quantum Electronics and Photonics Division, National Institute of Standards and Technology, \\ Boulder, Colorado 80305
}

Superconducting electronics play an important role in quantum computation, ultra-low-power electronics, voltage standards and magnetic sensors. Many of these applications rely on the microfabrication of superconducting circuits with multiple wiring layers and a range of active elements. The performance of these superconducting circuits are directly related to the three-dimensional microstructures of these wiring layers and active elements. Focused-ion-beam tomography is an ideal tool for exploring this microstructure, however the processes of milling, imaging, and reconstruction must be optimized for the information most pertinent to superconducting electronics. We present the optimization of tomographic collection and reconstruction using the NIST designed 10 V Programmable Josephson Voltage Standard (PJVS).

The PJVS designed at NIST consists of 268,800 Josephson junctions that are microwave-biased at approximately $18 \mathrm{GHz}$ [1]. The PJVS is designed to optimize the total voltage, microwave performance and operational stability. To accommodate the large number of junctions on a $12 \mathrm{~mm} \times 17 \mathrm{~mm}$ chip, junctions must be vertically stacked; however, the maximum stacking is limited by the maximum current capacity and yield considerations. Each stack normally consists of 3 junctions with a $\mathrm{Nb}_{\mathrm{x}} \mathrm{Si}_{\mathrm{I}} \mathrm{-}_{\mathrm{x}}$ layer creating a normal-metal tunnel barrier. Each of these stacks is series connected using wiring layers of $\mathrm{Nb}$ above or below the stack using vias through the electrically insulating layer. Structural integrity, packaging, and electrical isolation are all achieved using $\mathrm{SiO}_{2}$ layers of different thicknesses. The device is supported on a $\mathrm{Si}(100)$ wafer for microfabrication compatibility.

Devices to be analyzed were first epoxied or carbon taped to standard $12.7 \mathrm{~mm}$ aluminum SEM stubs. If the devices had $\mathrm{SiO}_{2}$ for passivation, then coating with a thin layer of $\mathrm{Pt}(\sim 40 \mathrm{~nm})$ was selected for charge dissipation. It was discovered that preparation of the sample for tomograms was best if the samples were firmly affixed with conductive epoxy, coated with Pt and the coating of Pt was electrically connected to the stub. The samples were milled and then imaged in a FIB-FESEM . In order to optimize tomography, the effects of milling current, milling protection layers, and imaging parameters were explored. In addition to the optimization of milling and imaging parameters, $3 \mathrm{D}$ reconstructions were performed using post-processing tools.

For the most efficient collection of tomograms, it is important to select the Ga milling current that reveals the features of interest at the highest rate. The milling current was varied from $16 \mathrm{nA}$ to $20 \mathrm{pA}$ and resulting single cross-sections were analyzed for mill face roughness or "curtaining". The mill face roughness decreases with current until $120 \mathrm{pA}$, at which point it remains constant at a value of $\sim 18 \mathrm{~nm}$ $\pm 6 \mathrm{~nm}$. Similarly, it is important to choose imaging parameters that provide high contrast and sufficient resolution of the features of interest in the shortest time possible. With a large number of detector, detector bias, electron acceleration voltages and electron beam currents, the selection of optimal parameters requires careful evaluation. Three detectors with selectable biases are available for fast tomogram imaging: an in-lens detector, sensitive to low-energy secondary electrons, an EverhartThornley type detector, sensitive to both low- and high-energy electrons at a variety of angles, and an 
in-lens energy selective backscatter detector, sensitive to high angle, high energy electrons. The in-lens detector provides the highest contrast and resolution; however, its sensitivity to accumulated charge in the oxide layer causes large changes in the contrast of the image during acquisition, rendering it nonoptimal. The in-lens energy-selective backscatter detector has lower detection efficiencies and lower resolution, requiring longer integration times and providing images of insufficient quality. Finally the Everhart-Thornley-type detector provides a compromise leading to images of sufficiently high quality in relatively short integration times. The electron beam aperture and condenser lens setting are chosen to provide an image resolution greater than the mill-face surface-roughness in the smallest time with the highest depth of field (60 um aperture, low condenser excitation). (See Table 1) Data are then collected by milling a slice, imaging with the Ga beam deactivated, and repeating for the full volume of interest.

Once a set of slices is acquired, post-acquisition image processing is applied to create three dimensional reconstructions of the data set. Image brightness is used to select features and materials of interest. A mask for the images is created by a high- and low-brightness threshold, and then the brightness information within these bounds is retained. Each element in the stack is then selectively cropped, accounting for the motion of the mill face. Small drifts are accounted for by feature recognition of unmilled areas on the surface of the sample and removed by adjustment of the selective cropping window. The resultant slices, now corrected for drift and brightness-selected, are rendered in three dimensions by use of free software.

\section{References:}

[1] P. D. Dresselhaus et al, IEEE Transactions on Applied Superconductivity 21 no 3. (2011) p. 693-696

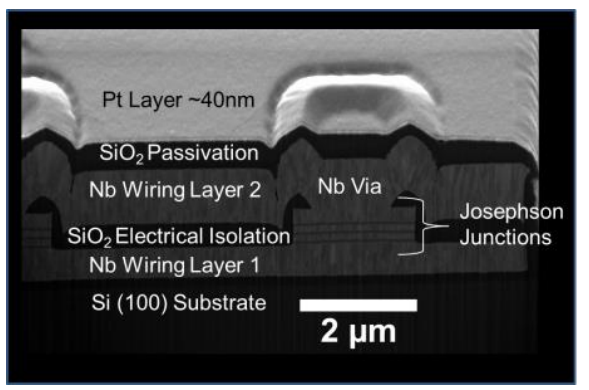

\begin{tabular}{|l|l|}
\hline Parameter & Optimal Value \\
\hline Charge Dissipation & $\begin{array}{l}40 \mathrm{~nm} \text { Pt Layer Electrically Connected } \\
\text { to Conductive Epoxy on Stub }\end{array}$ \\
\hline Gallium Ion Milling Current & $120 \mathrm{pA}$ \\
\hline Electron Imaging Current* & $480 \mathrm{pA}$ \\
\hline Imaging Acceleration Voltage & $5 \mathrm{kV}$ \\
\hline Image Pixel Dwell Time & $1.3 \mu \mathrm{s}$ \\
\hline Imaging Detector & Everhart-Thornley \\
\hline Slice Imaging Time & $10.2 \mathrm{~s}$ \\
\hline
\end{tabular}

Figure 1. Superconducting Test Structure and Optimized Milling and Imaging Parameters. Typical cross-sectional view of a $2 \mu \mathrm{m}$ superconducting-normal-superconducting junction stack, electrical isolation and wiring layers including the parameters found to be optimal for milling and imaging.

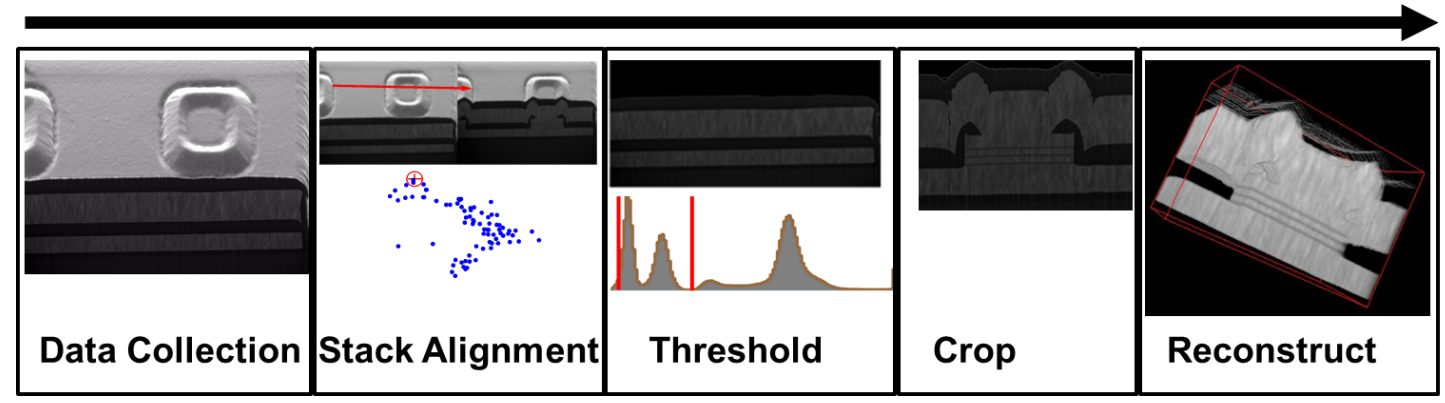

Figure 2. 3D Reconstruction Flow. The linear process of $3 \mathrm{D}$ reconstruction of the $2 \mu \mathrm{m}$ junction stack featured in Figure 1. 